\title{
Tunable Miscibility in a Dual-Species Bose-Einstein Condensate
}

\author{
S. B. Papp, ${ }^{1, *}$ J. M. Pino, ${ }^{1}$ and C. E. Wieman ${ }^{2,1}$ \\ ${ }^{1}$ JILA, National Institute of Standards and Technology and University of Colorado, Boulder, Colorado 80309-0440, USA \\ ${ }^{2}$ University of British Columbia, Vancouver, BC V6T 1Z1, Canada
}

(Received 19 February 2008; published 24 July 2008)

\begin{abstract}
We report on the observation of controllable phase separation in a dual-species Bose-Einstein condensate with ${ }^{85} \mathrm{Rb}$ and ${ }^{87} \mathrm{Rb}$. Interatomic interactions between the different components determine the miscibility of the two quantum fluids. In our experiments, we can clearly observe immiscible behavior via a dramatic spatial separation of the two species. Furthermore, a magnetic-field Feshbach resonance is used to change them between miscible and immiscible by tuning the ${ }^{85} \mathrm{Rb}$ scattering length. The spatial density pattern of the immiscible quantum fluids exhibits complex alternating-domain structures that are uncharacteristic of its stationary ground state.
\end{abstract}

DOI: 10.1103/PhysRevLett.101.040402

Two-component quantum fluids exhibit rich physics that is not accessible in a single-component fluid. Phase separation of the two components is a particularly dramatic phenomenon that was observed long ago in ${ }^{3} \mathrm{He}^{-}{ }^{4} \mathrm{He}$ mixtures [1]. Ultracold quantum gases also provide a unique opportunity to study the miscibility of interpenetrating quantum fluids. They exhibit numerous advantages including the resonant control of two-body interactions via magnetic-field Feshbach resonances, which provides a parameter to directly alter the miscibility of the fluids.

The first two-component condensate was produced with different hyperfine states of ${ }^{87} \mathrm{Rb}$, and evidence for repulsive interactions between the two clouds was reported [2]. The dynamical behavior of a two-component BoseEinstein condensate (BEC) has been studied extensively $[3,4]$ including the dramatic observation of weakly damped collective oscillations [5]. In spinor condensates both miscible and immiscible two-component condensates were observed for the first time [6]. They formed long-lived metastable excited states composed of magnetic domains [7], and tunneling effects between the domains were studied [8]. Dual-species quantum gases are currently a subject of significant interest. In particular, an outstanding goal is the creation of ultracold heteronuclear molecules in low-lying vibrational states. Such molecules are expected to possess a dipole moment that could be utilized for such varied applications as quantum information and the search for the electron electric dipole moment [9]. A dual-species BEC of ${ }^{41} \mathrm{~K}$ and ${ }^{87} \mathrm{Rb}$ has already been realized in a harmonic potential [10] and in an optical lattice [11]. In a Bose-Fermi mixture of ${ }^{40} \mathrm{~K}$ and ${ }^{87} \mathrm{Rb}$, a tunable interspecies scattering length was used to probe both phase separation of the mixture and interaction-induced collapse [12].

Understanding how the spatial overlap of a dual-species BEC changes as interactions are tuned will be important for future experiments. In this Letter, we explore the tunable miscibility of a Bose-condensed mixture of ${ }^{85} \mathrm{Rb}$ and ${ }^{87} \mathrm{Rb}$ gases. Immiscibility of the two-component quantum gas is observed as a dramatic departure of the density distribution from the symmetry of the trapping potential.
PACS numbers: 03.75.Mn, 03.75.Hh, 05.30.Jp

Surprisingly, we observe the robust formation of multiple, nonoverlapped, single-species BEC "cloudlets" that represent an interesting presumably metastable excited state.

Theoretical investigations of two-component condensates have illuminated the role that interatomic interactions play in determining the density patterns and phase separation of the components [13-17]. Spatial separation of two immiscible quantum fluids in a trap is typified by a balland-shell ground-state structure in which one fluid forms a low density shell around the other. This structure depends on the relative strength of interspecies and single-species interactions. Other ground-state patterns are expected as well, and a general classification of all possible ground states has been presented in Ref. [18]. A mixture of ${ }^{85} \mathrm{Rb}$ and ${ }^{87} \mathrm{Rb}$ has long been considered a promising experimental system for studying two interpenetrating quantum fluids. The tendency for spatial separation to occur in a ${ }^{85} \mathrm{Rb}_{-}{ }^{87} \mathrm{Rb}$ mixture can be predicted by applying the theoretical analysis of Ref. [19] for the Thomas-Fermi limit. We define the parameter $\Delta=\frac{a_{85} a_{87}}{a_{85-87}^{2}}-1$ that depends upon the ratio of the single-species and interspecies interactions. In the case where $a_{85-87}$ is positive, two regimes emerge: $\Delta>0$, where the two condensates are miscible, and $\Delta<$ 0 , where they are immiscible due to the strong interspecies repulsion. For simplicity, we have neglected the small difference in mass of ${ }^{85} \mathrm{Rb}$ and ${ }^{87} \mathrm{Rb}$.

Our apparatus has been described previously [20]. Atoms are initially collected from a vapor into a twospecies magneto-optical trap (MOT). After transfer to another region with lower vacuum pressure, the atoms are prepared in the ${ }^{85} \mathrm{Rb}\left|f=2, m_{f}=-2\right\rangle$ state and ${ }^{87} \mathrm{Rb}\left|f=1, m_{f}=-1\right\rangle$ state and loaded into a magnetic trap. Selective rf evaporation is used to lower the temperature of the ${ }^{87} \mathrm{Rb}$ gas to $10 \mu \mathrm{K}$, while the ${ }^{85} \mathrm{Rb}$ gas is sympathetically cooled. The two species are then loaded into an optical dipole trap [Fig. 1] while maintaining a large, nearly uniform magnetic field. Further evaporation to BEC is performed by lowering the depth of the optical potential. Unless otherwise noted, after evaporation the depth of the 
optical trap is raised in $50 \mathrm{~ms}$ to $\sim 1 \mu \mathrm{K}$ with a measured ${ }^{85} \mathrm{Rb}$ radial (axial) trap frequency of $2 \pi \times 130 \mathrm{~Hz}(2 \pi \times$ $2.9 \mathrm{~Hz}$ ). While the ${ }^{87} \mathrm{Rb}$ radial frequency is nearly identical to that of ${ }^{85} \mathrm{Rb}$, the axial frequency is smaller $(2 \pi \times$ $2.6 \mathrm{~Hz}$ ) due to a different magnetic moment.

To create a ${ }^{85} \mathrm{Rb} \mathrm{BEC}$, it is important to use a weakly confining optical trap to reduce density-dependent inelastic loss and to control the scattering length of ${ }^{85} \mathrm{Rb}$ using the Feshbach resonance. Specifically, condensates are created only in a small range of magnetic field near $163.1 \mathrm{G}$, where the ${ }^{85} \mathrm{Rb}$ interaction is repulsive [21,22] and inelastic collision rates are sufficiently small. By optimizing the evaporation rate and the precise number ratio of the two species at the start of evaporation, we are able to create a singlespecies ${ }^{85} \mathrm{Rb}$ BEC with up to $8 \times 10^{4}$ atoms, a ${ }^{87} \mathrm{Rb}$ BEC with $2 \times 10^{6}$ atoms, or a BEC of both species. Absorption images of single-species condensates after expansion are shown in Fig. 1. In this Letter, the elongated axial direction (z) of all of the images shows the spatial density of the gas since the trapping frequency is small. The radial direction shows the momentum density. Several interspecies Feshbach resonances are accessible in our system including the $s$-wave resonances used in Ref. [20] as well as recently discovered $p$-wave resonances at $257.8 \mathrm{G}$ and $\approx 330 \mathrm{G}$ [23]. For the magnetic fields used in this work, however, the interspecies scattering length is $+213(7) a_{0}$ [24], and the ${ }^{87} \mathrm{Rb}$ scattering length is $+99 a_{0}$ [25].
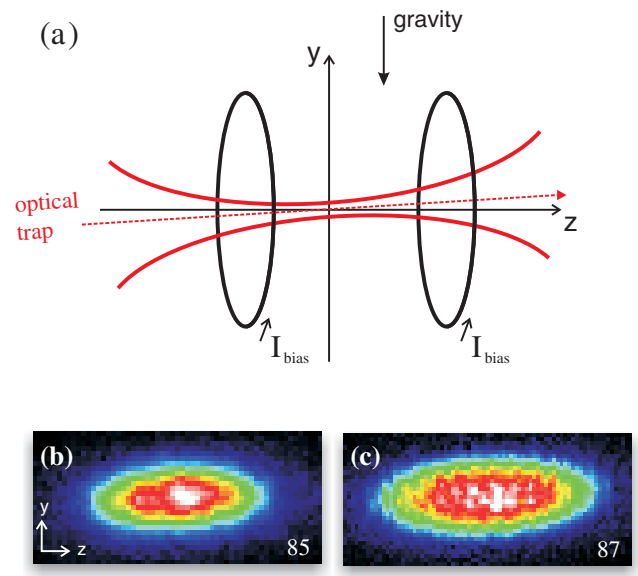

FIG. 1 (color online). (a) Schematic drawing of the optical trap laser beam and the magnetic-field coils. The curvature of the magnetic field enhances confinement along the axial direction (z) of the optical trap. The center position of the trapping potential along its axis is influenced by the tilt of the optical trap relative to gravity and the relative position of the optical trap beam focus and the coils. Optical trap tilt and misalignment of the beam focus and the coils have been exaggerated for clarity. Absorption images are acquired along the axis that is into the page. (b),(c) Absorption images of single-species ${ }^{85} \mathrm{Rb}$ and ${ }^{87} \mathrm{Rb}$ condensates demonstrate a smooth density profile, consistent with the shape of the trapping potential. Prior to imaging, the gas is released from the optical trap and expands for $20 \mathrm{~ms}$; the magnetic field remains on for the first $10 \mathrm{~ms}$ of expansion to prevent collapse of the ${ }^{85} \mathrm{Rb}$ gas.
Interesting physics occurs when we create a simultaneously Bose-condensed sample of ${ }^{85} \mathrm{Rb}$ and ${ }^{87} \mathrm{Rb}$. The dual BEC exhibits either miscible or immiscible behavior, depending on the magnetic field, as seen in Fig. 2. After evaporative cooling, the magnetic field was swept in $400 \mathrm{~ms}$ from $163.1 \mathrm{G}$ to a value in the range of 164.6$158.6 \mathrm{G}$; the sweep covered a range in $\Delta$ of approximately -1 to $+1\left(50 a_{0}-900 a_{0}\right)$. The rate was measured to be slow with respect to the response of the gases in the radial and axial directions. Images of each gas were acquired after the sweep and $20 \mathrm{~ms}$ of expansion from the trap. They are shown for $\Delta=-0.89$ in Figs. 2(a) and 2(b) and $\Delta=$ 0.72 in Figs. 2(c) and 2(d). Summing the radial direction of the images reveals the position-space distribution [Figs. 2(e) and 2(f)] since little expansion occurs along the trap axis. We observe a dramatic spatial separation of the two gases when $\Delta$ is negative. The irregular spikes in
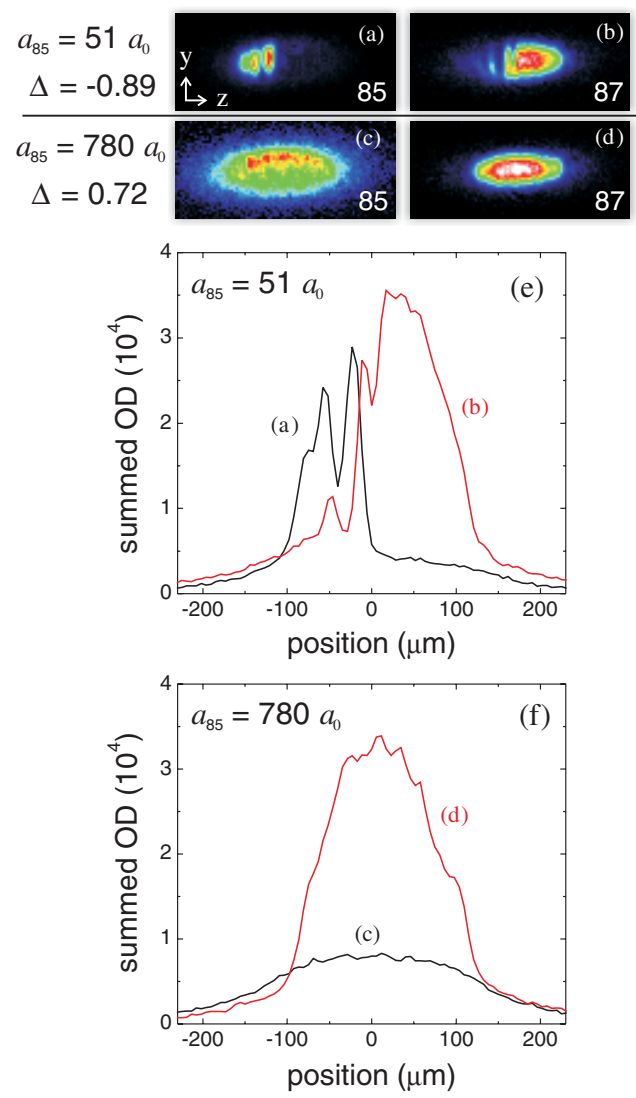

FIG. 2 (color online). Absorption images at two different ${ }^{85} \mathrm{Rb}$ scattering lengths that display (a),(b) immiscible and (c),(d) miscible behavior. Image (c) has been scaled by a factor of 5 for clarity. Each image in the pairs (a),(b) and (c),(d) was acquired with different condensates after creation under the same conditions. The sum of each pair should be interpreted as the total density of the expanded gas. (e),(f) The measured twodimensional optical density (OD) was summed in the remaining radial direction to produce a cross section. The ${ }^{85} \mathrm{Rb}$ scattering length was $51 a_{0}$ for images (a) and (b) and it was $780 a_{0}$ for images (c) and (d). The number of ${ }^{85} \mathrm{Rb}\left({ }^{87} \mathrm{Rb}\right)$ atoms is $4.5 \times$ $10^{4}\left(9 \times 10^{4}\right)$. 
the density patterns of the two condensates will be discussed in more detail below. When $\Delta$ is positive, the coexistence of each species throughout the axial direction is observed, and the sharp density spikes of the immiscible condensates give way to a smooth overall density. Spatial separation was observed to return when the magnetic-field sweep to $\Delta>0$ was immediately followed by a second opposite field sweep to $\Delta<0$. The penetration distance in the axial direction over which the two expanded gases overlap is estimated to be approximately $30 \mu \mathrm{m}$ when $\Delta=-0.89$.

To characterize the degree of spatial separation as $\Delta$ is varied, we have extracted the axial center-of-mass location of each species from images similar to those shown in Fig. 2. In this experiment, an offset between the axial trap centers for each species was intentionally introduced [Fig. 1(a)] to reflect the spatial separation in the center-ofmass location. The offset causes the two immiscible quantum gases to separate primarily in the axial direction with each species preferring a well-defined side of the trap. The results of this experiment are plotted in Fig. 3 as a function of $\Delta$. The centers of each BEC gradually come together as $\Delta$ is increased from -0.9 toward zero. For $\Delta>0$ there is little change as the two species can then coexist throughout the trap. However, residual gravitational sag likely induces incomplete spatial overlap even when $\Delta>0$.

We have studied the different immiscible density patterns as a function of the number of each $\mathrm{Rb}$ species. We vary the relative number in the two-species MOT to change the number of atoms in the BECs. Figure 4 shows images of both ${ }^{85} \mathrm{Rb}$ and ${ }^{87} \mathrm{Rb}$ condensates immediately after evaporation to a fixed final trap depth of $\approx 100 \mathrm{nK}$, and $\Delta=-0.82\left(81 a_{0}\right)$ so that the two quantum fluids are always immiscible. In each image, a different condensate is shown since the measurement process is destructive. The

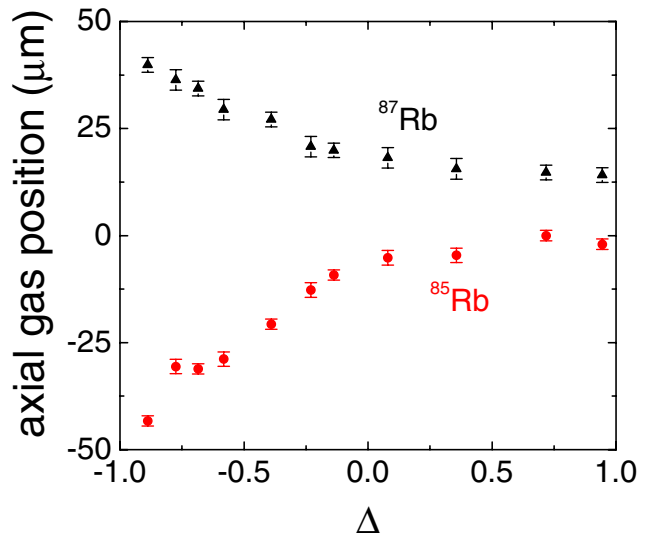

FIG. 3 (color online). Measured axial position of the ${ }^{85} \mathrm{Rb}$ and ${ }^{87} \mathrm{Rb}$ gases as a function of the parameter $\Delta$. We report the uncertainty in the position as the shot-to-shot reproducibility of our measurements. When $\Delta>0$, the measured difference in the position of each gas is in good agreement with that expected for single-species condensates. The number of ${ }^{85} \mathrm{Rb}\left[{ }^{87} \mathrm{Rb}\right]$ atoms is $5.0(5) \times 10^{4}\left[1.2(2) \times 10^{5}\right]$. trap was not recompressed in order to accentuate the spatial separation of the two species.

When a condensate of each species is present in the trap, we observe "holes" in the ${ }^{87} \mathrm{Rb}$ image that correspond to "spikes" at approximately the same location in the ${ }^{85} \mathrm{Rb}$ image [Figs. 4(a)-4(d)]. This behavior is consistent with spatial separation of two immiscible quantum fluids. However, the images are not consistent with any known phase-separation structure expected for the ground state of a two-component system as described in Ref. [18]. The ${ }^{85} \mathrm{Rb}$ gas is observed to split into multiple separated cloudlets that alternate with distinct holes in the ${ }^{87} \mathrm{Rb}$ gas. In Figs. 4(a) and 4(b), two ${ }^{85} \mathrm{Rb}$ cloudlets are observed, and three cloudlets are observed in Fig. 4(c). The total density of the gas, found by summing corresponding ${ }^{85} \mathrm{Rb}$ and ${ }^{87} \mathrm{Rb}$ images, mostly preserves the symmetry of the trap. These images represent long-lived presumably metastable excited states of the two-component system that depend on the relative number of each species. If the initial number of ${ }^{85} \mathrm{Rb}$ atoms at the start of evaporation is too large, the ${ }^{87} \mathrm{Rb}$ gas cannot be cooled to condensation and remains a dilute thermal gas; in this case [Fig. 4(e)] the ${ }^{85} \mathrm{Rb}$ condensate
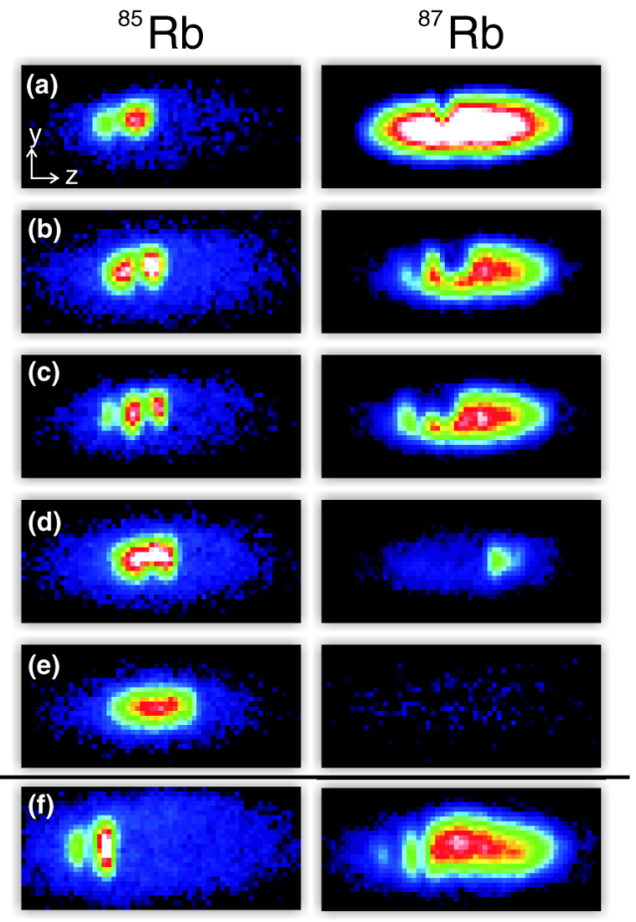

FIG. 4 (color online). (a)-(e) Absorption images of ${ }^{85} \mathrm{Rb}$ (left column) and ${ }^{87} \mathrm{Rb}$ (right column) condensates demonstrating the immiscibility of the quantum gases. For clarity, the OD of each ${ }^{87} \mathrm{Rb}$ image was scaled by a factor of 0.5 , and the size of each image is $330 \mu \mathrm{m} \times 150 \mu \mathrm{m}$. The number of ${ }^{85} \mathrm{Rb}$ particles varies from approximately $1.2 \times 10^{4}$ in (a) to $2.2 \times 10^{4}$ in (e); the ${ }^{87} \mathrm{Rb}$ number varies from $1.4 \times 10^{5}$ in (a) to $3 \times 10^{3}$ in (e). For comparison, images of single-species ${ }^{85} \mathrm{Rb}$ and ${ }^{87} \mathrm{Rb}$ condensates are shown in Figs. 1(b) and 1(c). (f) Absorption images following recompression of the optical trap that demonstrate a more symmetrical radial distribution. 
shape is unperturbed and well approximated by a ThomasFermi model.

The immiscible gases shown in Fig. 4 are not symmetric with respect to either the radial or the axial directions. Gravitational sag induces a small offset between the minima of the traps [Fig. 1] for the two clouds leading to the observed asymmetries. The density pattern of a twocomponent BEC including an offset has been studied theoretically, and a separation of the cloud centers is expected [19]. The asymmetries can be reduced by recompression to increase the trap depth [Fig. 4(f)]. Symmetry in the radial direction is mostly restored, but spatial separation is still clearly observed along the axial dimension. Similarly, we have verified that the axial offset can be controlled by aligning the optical trap focus with respect to the magnetic field [Fig. 1(a)]. Although the precise shape, size, and position of the immiscible gases are affected by details of the trapping potential, the observation of spatial separation and the formation of separated ${ }^{85} \mathrm{Rb}$ cloudlets is repeatable under a variety of experimental conditions including the rate of evaporation, the final trap depth, and the ${ }^{85} \mathrm{Rb}$ scattering length during evaporation. The spatial separation and cloudlet behavior are observed during the cooling process as soon as the temperature of both species is lowered below the condensation temperature. Furthermore, the dual-species BEC remains spatially separated, and individual cloudlets of ${ }^{85} \mathrm{Rb}$ do not coalesce during the $1 \mathrm{~s}$ lifetime of the ${ }^{85} \mathrm{Rb} \mathrm{BEC}$, which includes many radial and a few axial trap oscillations.

We lack a detailed theoretical understanding of ${ }^{85} \mathrm{Rb}$ cloudlet formation in our experiments. The geometry of our system is complicated by the elongated axial direction of the trap and the presence of gravitational sag. In an analysis of the favorability for cloudlet formation, the energy cost of forming and maintaining extra boundary surface area must be weighed against the cost of all particles residing in the same cloud [16]. These questions appear to require the solution of coupled Gross-Pitaevskii equations for the dual-species BEC wave functions, which is beyond the scope of this Letter [14]. The formation of cloudlets may be due to a modulation instability, in which weak perturbations of a nonlinear system grow rapidly into high-contrast spatial density waves [26]. Numerical simulations of two-component BECs [27] bear a strong resemblance to the images in Fig. 4. Specifically, the observation of alternating-domain structures that preserve the total density and persist for many trap oscillation periods matches the theoretical prediction. Recent theoretical simulations specific to our system have obtained qualitative agreement with the data presented here [28].

In summary, we have produced a dual-species BEC of ${ }^{85} \mathrm{Rb}$ and ${ }^{87} \mathrm{Rb}$ with tunable interactions. Immiscibility of the two quantum fluids was observed as a departure in the gas density pattern from that expected due to the symmetry of the potential. Surprisingly, immiscible condensates are observed to form spatially separated cloudlets. Numerous
Feshbach resonances are available in the ${ }^{85} \mathrm{Rb}^{8}{ }^{87} \mathrm{Rb}$ system, making it possible to study a two-component condensate with a tunable interspecies interaction.

We gratefully acknowledge useful discussions with Shai Ronen, Mark Edwards, Deborah Jin, and Eric Cornell. This work has been supported by NSF and ONR.

Note added.-Recently, we learned of similar work reported in Ref. [29].

*Present address: Norman Bridge Laboratory of Physics 12-33, California Institute of Technology, Pasadena, CA 91125, USA.

[1] G. K. Walters and W. M. Fairbank, Phys. Rev. 103, 262 (1956); E. H. Graf, D. M. Lee, and J. D. Reppy, Phys. Rev. Lett. 19, 417 (1967).

[2] C. J. Myatt et al., Phys. Rev. Lett. 78, 586 (1997).

[3] D. S. Hall et al., Phys. Rev. Lett. 81, 1539 (1998).

[4] H. Lewandowski et al., Phys. Rev. Lett. 88, 070403 (2002).

[5] K. M. Mertes et al., Phys. Rev. Lett. 99, 190402 (2007).

[6] J. Stenger et al., Nature (London) 396, 345 (1998).

[7] H.-J. Miesner et al., Phys. Rev. Lett. 82, 2228 (1999).

[8] D. M. Stamper-Kurn et al., Phys. Rev. Lett. 83, 661 (1999).

[9] J. J. Hudson, B. E. Sauer, M. R. Tarbutt, and E. A. Hinds, Phys. Rev. Lett. 89, 023003 (2002).

[10] G. Modugno et al., Phys. Rev. Lett. 89, 190404 (2002).

[11] J. Catani et al., Phys. Rev. A 77, 011603(R) (2008).

[12] C. Ospelkaus et al., Phys. Rev. Lett. 97, 120402 (2006).

[13] T.-L. Ho and V.B. Shenoy, Phys. Rev. Lett. 77, 3276 (1996).

[14] B. D. Esry, C. H. Greene, J. P. Burke, Jr., and J. L. Bohn, Phys. Rev. Lett. 78, 3594 (1997).

[15] P. Ao and S. T. Chui, Phys. Rev. A 58, 4836 (1998).

[16] E. Timmermans, Phys. Rev. Lett. 81, 5718 (1998).

[17] H. Pu and N. P. Bigelow, Phys. Rev. Lett. 80, 1130 (1998).

[18] M. Trippenbach et al., J. Phys. B 33, 4017 (2000).

[19] F. Riboli and M. Modugno, Phys. Rev. A 65, 063614 (2002); D. M. Jezek and P. Capuzzi, Phys. Rev. A 66, 015602 (2002).

[20] S. B. Papp and C. E. Wieman, Phys. Rev. Lett. 97, 180404 (2006).

[21] S. L. Cornish et al., Phys. Rev. Lett. 85, 1795 (2000).

[22] N. R. Claussen et al., Phys. Rev. A 67, 060701(R) (2003).

[23] C. Ticknor, C. A. Regal, D. S. Jin, and J. L. Bohn, Phys. Rev. A 69, 042712 (2004).

[24] J. P. Burke and J. L. Bohn, Phys. Rev. A 59, 1303 (1999).

[25] E. G. M. van Kempen, S. J. J. M. F. Kokkelmans, D. J. Heinzen, and B. J. Verhaar, Phys. Rev. Lett. 88, 093201 (2002).

[26] K. Kasamatsu and M. Tsubota, Phys. Rev. Lett. 93, 100402 (2004).

[27] K. Kasamatsu and M. Tsubota, J. Low Temp. Phys. 138, 669 (2005).

[28] S. Ronen, M. Edwards, and J. L. Bohn (unpublished).

[29] G. Thalhammer et al., Phys. Rev. Lett. 100, 210402 (2008). 